UCRL-JC-121494

PREPRINT

\title{
Laser Plasma Diagnostics of Dense Plasmas
}

S. G. Glendinning, P. Amendt, K. S. Budil, B. A. Hammel D. H. Kalantar, M. H. Key, O. L. Landen, B. A. Remington, D. E. Desenne

This paper was prepared for submittal to the SPIE Conference on Applications of Laser Plasma Radiation II, San Diego, CA

July 9-14, 1995

July 12, 1995

This is a preprint of a paper intended for publication in a journal or proceedings. Since changes may be made before publication, this preprint is made available with the understanding that it will not be cited or reproduced without the permission of the author. 


\section{DISCLAIMER}

This document was prepared as an account of work sponsored by an agency of the United States Government. Neither the United States Government nor the University of California nor any of their employees, makes any warranty, express or implied, or assumes any legal liability or responsibility for the accuracy, completeness, or usefulness of any information, apparatus, product, or process disclosed, or represents that its use would not infringe privately owned rights. Reference herein to any specific commercial product, process, or service by trade name, trademark, manufacturer, or otherwise, does not necessarily constitute or imply its endorsement, recommendation, or favoring by the United States Government or the University of California. The views and opinions of authors expressed herein do not necessarily state or reflect those of the United States Government or the University of California, and shall not be used for advertising or product endorsement purposes. 


\section{DISCLAIMER}

Portions of this document may be illegible in electronic image products. Images are produced from the best available original document. 
Laser plasma diagnostics of dense plasmas

\author{
S.G.Glendinning, P.Amendt, K.S.Budil, B.A.Hammel, D.H.Kalantar, M.H.Key", O.L.Landen, \\ B.A.Remington, D.E.Desenne* \\ Lawrence Livermore National Laboratory, P.O. Box 5508, Livermore, CA 94550 US \\ \# On leave at LLNL; Rutherford Appleton Lab., UK; University of Oxford, UK \\ * CEL-V, Limeil, FR
}

\begin{abstract}
We describe several experiments on Nova that use laser-produced plasmas to generate $x$-rays capable of backlighting dense, cold plasmas $\left(\rho \sim 1-3 \mathrm{gm} / \mathrm{cm}^{3}, \mathrm{kT} \sim 5-10 \mathrm{eV}\right.$, and areal density $\left.\rho \ell \sim 0.01-0.05 \mathrm{~g} / \mathrm{cm}^{2}\right)$. The $\mathrm{x}-$ rays used vary over a wide range of $h v$, from $80 \mathrm{eV}$ ( (X-ray laser) to $9 \mathrm{keV}$. This allows probing of plasmas relevant to many hydrodynamic experiments. Typical diagnostics are 100 ps pinhole framing cameras' for a long pulse backlighter and a time-integrated CCD camera for a short pulse backlighter.
\end{abstract}

\title{
Introduction
}

Inertial confinement fusion requires that high density plasmas be achieved, whether by compressing fuel directly with laser light or indirectly with $x$-rays in a cavity. Characterizing such plasmas and understanding the hydrodynamics relevant to implosions has been one of the goals of the Nova laser, and for several years ${ }^{2}$ we have used laser produced plasmas as a source of $\mathrm{x}$-rays capable of probing dense plasmas.

\section{Spectra}

We have measured spectra from absolutely calibrated crystal spectrometers and $x$-ray diodes to determine spectra of the various backlighters we use. We generate these with an incident laser intensity of between 3 and $10 \times 10^{14} \mathrm{~W} / \mathrm{cm}^{2}$ of $0.53 \mu \mathrm{m}$ light for $1-2 \mathrm{~ns}$ on solid planar targets. Some of these are shown in Figure 1 , for uranium, aluminum, molybdenum, scandium, titanium, and iron. (The error on the absolute measurement is $\pm 50 \%$ for both the crystal spectrometers and $x$-ray diode array; the relative error is $\pm 10 \%{ }^{3}$ ) While it is known ${ }^{4}$ that $0.35 \mu \mathrm{m}$ laser light (the third harmonic of Nova's $1.06 \mu \mathrm{m}$ beams and the usual frequency for driving hohlraums) has a higher efficiency for conversion into $x$-rays in the $1-9 \mathrm{keV}$ range than 0.53 (green) light does, we found that green light was much easier to use and that the penalty in $x$-ray conversion efficiency was made up for in the increased conversion efficiency from $1 \mu \mathrm{m}$ light to $0.53 \mathrm{vs}$. $0.35 \mu \mathrm{m}$ light.

The frequency tripling (or doubling) crystals used on Nova convert at an efficiency of $\sim 35 \%$ typically to third harmonic and $45 \%$ to second harmonic for our pulse lengths. To achieve the higher laser intensities quoted above, we used two of Nova's 10 beams. With two beams, we typically use about $8 \mathrm{~kJ}$ of $0.53 \mu \mathrm{m}$ light in a $2 \mathrm{~ns}$ flat-topped pulse to backlight a hydrodynamics experiment. The reasons for the high energy requirement are several. First, we are using gated microchannel plate pinhole cameras with $10 \mu \mathrm{m}$ pinholes to achieve our spatial resolution. These have a solid angle $\sim 10^{-7} \mathrm{sr}$ and are used at a magnification of $\sim 10$ to minimize the resolution degradation of the microchannel plate. The gated $\mathrm{MCP}$ signal is $\sim 0.1-1 \mathrm{ph} / \mu \mathrm{m}^{2} / \mathrm{ns}$ under these conditions. Second, we are backlighting structures which are $0.5-1.0 \mathrm{~mm}$ in extent. Finally, there is a significant background of high energy $x$-rays produced by the drive laser beams incident on the hohlraum wall.

We have found that we cannot produce a uniform, smooth backlighting spot with Nova beams without some form of beam smoothing. We use random phase plates on our backlighter beams on the large area backlighter experiments. A comparison of $\mathrm{x}$-ray images from a uranium backlighter with and without a RPP is in Figure 2. 


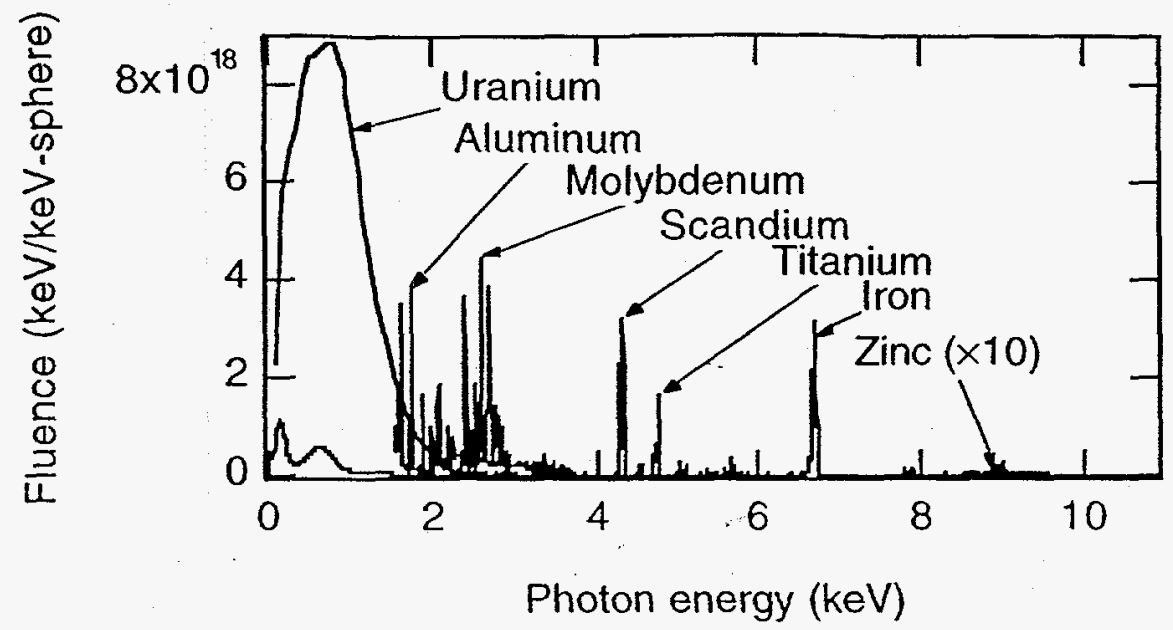

Figure 1. Measured spectra of laser-produced plasmas used for $\mathrm{x}$-ray backlighting experiments on Nova. Zn spectrum is from Ref .18 and is from a short pulse ( $100 \mathrm{ps})$ backlighter.
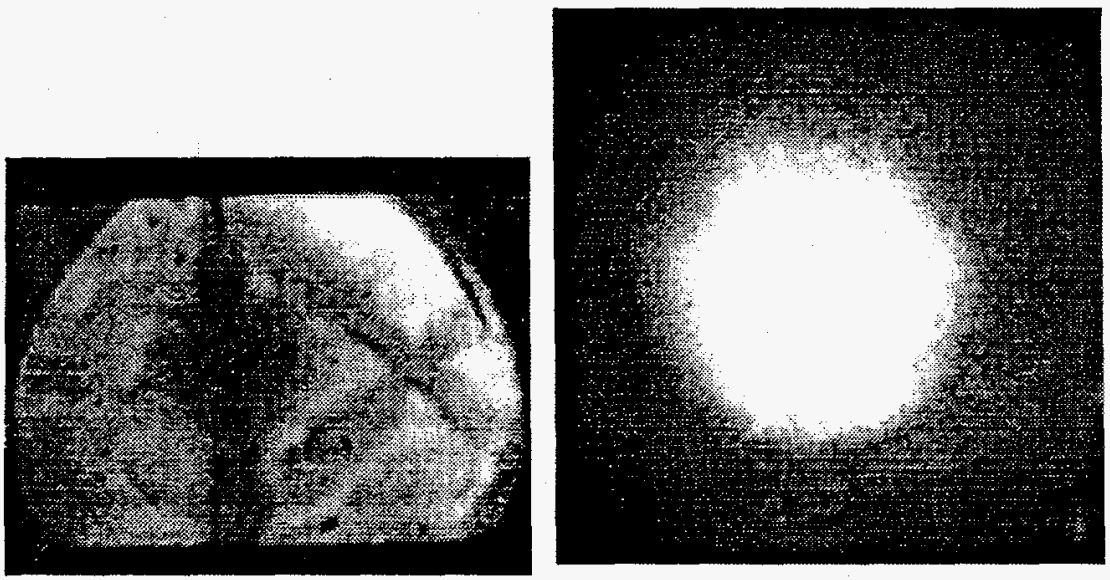

Figure 2. X-ray images of laser produced plasmas from uranium used for $x$-ray backlighting. The image on the left was produced by a beam without a random phase plate (RPP); structure in the laser beam reproduces in the $x$-ray spot. The image on the right used a RPP.

\section{Hydrodynamics experiments with large area backlighters}

On Nova we are currently pursuing several campaigns of hydrodynamics experiments using large area backlighters. These include: backlit implosions, time dependent drive symmetry measurements by foam witness balls, planar Rayleigh-Taylor, planar laser imprint experiments, and supernova relevant experiments. The last is of course not an ICF relevant experiment, yet it appears that the regimes of interest for astrophysical modeling may be studied on Nova.

Most of the large area backlighter hydrodynamics experiments on Nova use backlighters in the 4-6 keV range ( $\mathrm{Sc}, \mathrm{Ti}$, or $\mathrm{Fe}$, filtered with the same element at the diagnostic to select the He- $\alpha$ line). These backlighters produce $\sim 4 \times 10^{9}$ photons $/ \mu \mathrm{m}^{2} / \mathrm{sr} / \mathrm{ns}$ with an incident laser intensity of $5 \times 10^{14} \mathrm{~W} / \mathrm{cm}^{2}$. While a monochromatic spectrum makes analysis simpler, if a spectrum is well characterized it is not necessary to use a single line, 
especially if a medium $(\sim 1 \mathrm{keV})$ energy backlighter is desired. We have successfully used a uranium spectrum to backlight $20 \mu \mathrm{m}$ thick $\mathrm{CH}_{2}$ foils in agreement with simulations (as we describe below). We found that, at these laser intensities, candidate $\mathrm{K}$-shell backlighters such as $\mathrm{Mg}$ and $\mathrm{Al}$ had a significant contribution from bound-free continuum emission (see Figure 1) which was difficult to measure and which rendered them somewhat less than monochromatic.

\section{Backlit implosions}

The backlighting experiment which is the most directly related to the hydrodynamics of implosions is the backlit implosion itself. A series of implosions on Nova which are dedicated to understanding the effect of Rayleigh-Taylor growth on fuel temperature, convergence, and neutron yield uses dopant in the ablated pusher to maintain high pusher areal density (dopants prevent energetic $x$-rays in the drive from depositing in the pusher and lowering the density) ${ }^{5}$. Clearly, the pusher areal density is an important experimental parameter that is usually not measured but inferred from simulations. In a backlit implosion, the varying pusher areal density may be determined by the attenuation of the backlighter $x$-ray flux and compared with simulations. The experimental setup is shown in Figure 3 and a typical image is also shown in Figure 3.

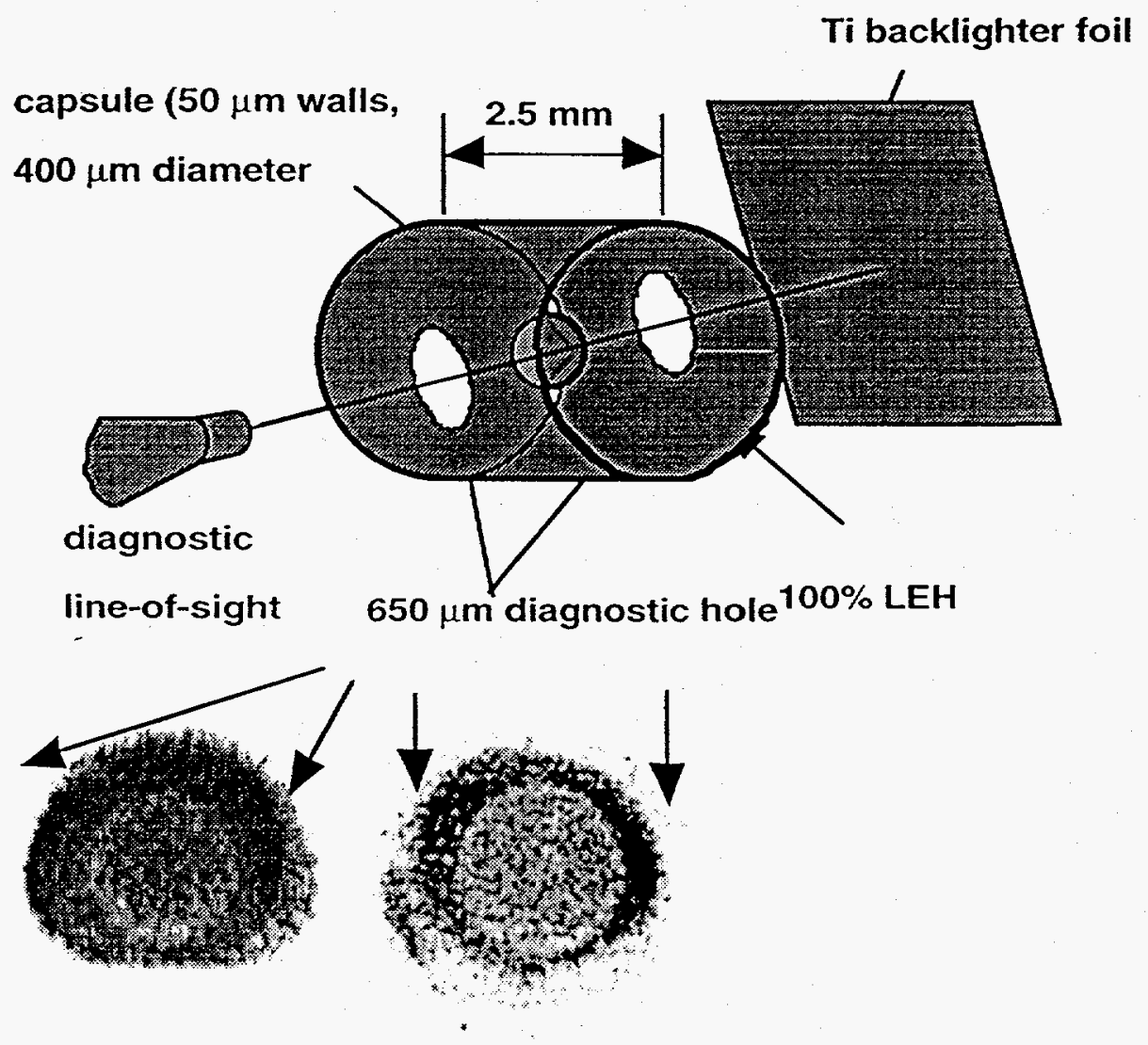

Figure 3. Experimental setup and typical images from backlit implosion and witness ball experiments. The pusher may be seen as a lighter ring (lower transmission) in the left hand image; in the image on the right a lighter ring is also visible but in this case it is due to the shock compression of the solid witness ball material. The $4.7 \mathrm{keV}$ Ti He- $\alpha$ backlighter is essentially unattenuated by the ablated material surrounding the capsule.

To determine pusher areal density, we perform an Abel inversion on an azimuthal average of each image to obtain the radial density profile as a function of time. Because of the high hv of the Ti He- $\alpha$ line used as a backlighter, the opacity does not depend on T or $\rho$. The resulting calculated optical depth is shown in Figure 4. 


\section{Time dependent drive symmetry by witness balls ${ }^{6}$}

Another application for backlit imaging in ICF experiments is the measurement of hohlraum drive symmetry from distortion of the induced shock in a low density ball. In such an experiment, the observable is the position of the shock front as a function of time and space in a sequence of images, determined from the absorption profile of the backlighter $x$-rays. For this experiment, the setup is essentially identical to that of the backlit implosions as shown in Figure 1, except that the imploding capsule is replaced with a low density $\left(\rho=0.3 \mathrm{gm} / \mathrm{cm}^{3}\right)$, solid, $\mathrm{SiO}_{2}$ ball of the same size as the capsule. The effect of the low density is to exaggerate the distortion of the pressure (and hence the shock) asymmetry:

$$
\frac{d r_{s}}{d t}=\left(\frac{\gamma+1}{2}\right)^{y / 2}\left(\frac{P_{a}}{\rho_{0}}\right)^{1 / 2}
$$

where $r_{s}$ is the position of the shock, $\gamma$ is the heat capacity, $P_{a}$ is the ablative shock, and $p_{0}$ is the density of the unshocked material. (However, too low a density can result in a transonic radiation wave preheating the ball and reducing the shock compression.) A typical image from this experiment is shown in Figure $4 \mathrm{~m}$ the right; in this case the ring of reduced transmission is due to shock compression of the foam, rather than the presence of a hollow shell as in the image of a capsule on the left. The shock is visible in azimuthal lineouts of frames at two different times (Figure 5) as a departure from the transmission profile expected for a sphere of constant density. Both the dip near $150 \mu \mathrm{m}$ radius and the rapid rise in transmission are features of the shock front; the ablation front is spatially separated from the shock front, especially later in time. We have used these witness balls successfully to determine distortions of a few percent.

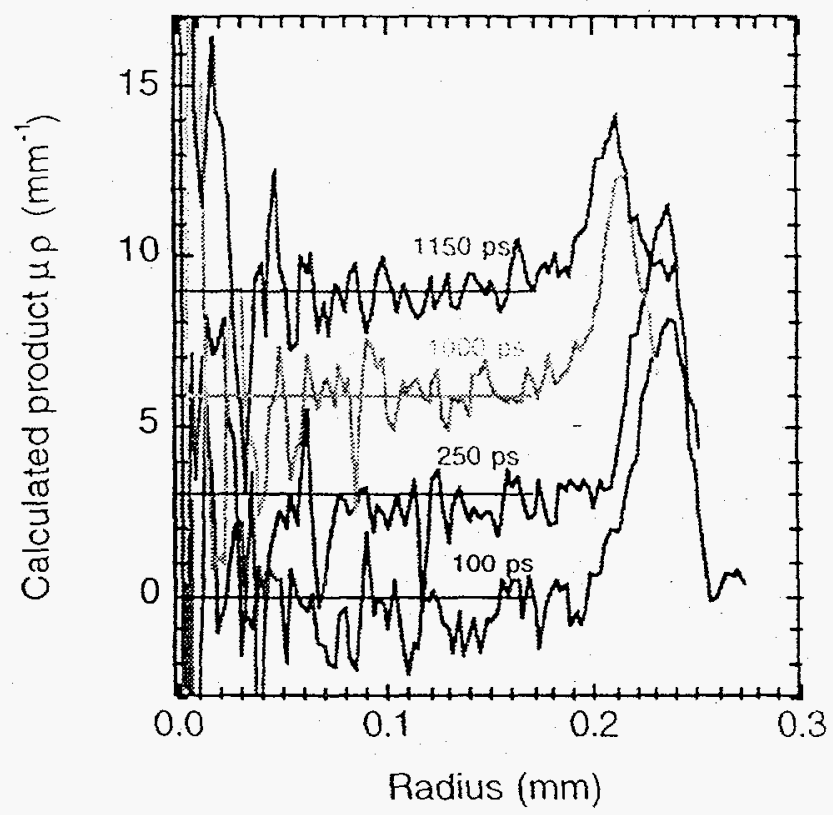

Figure 4. Abel inversion of azimuthal averages of a sequence of images from a backlit implosion. The lineouts are offset vertically for the illustration. 


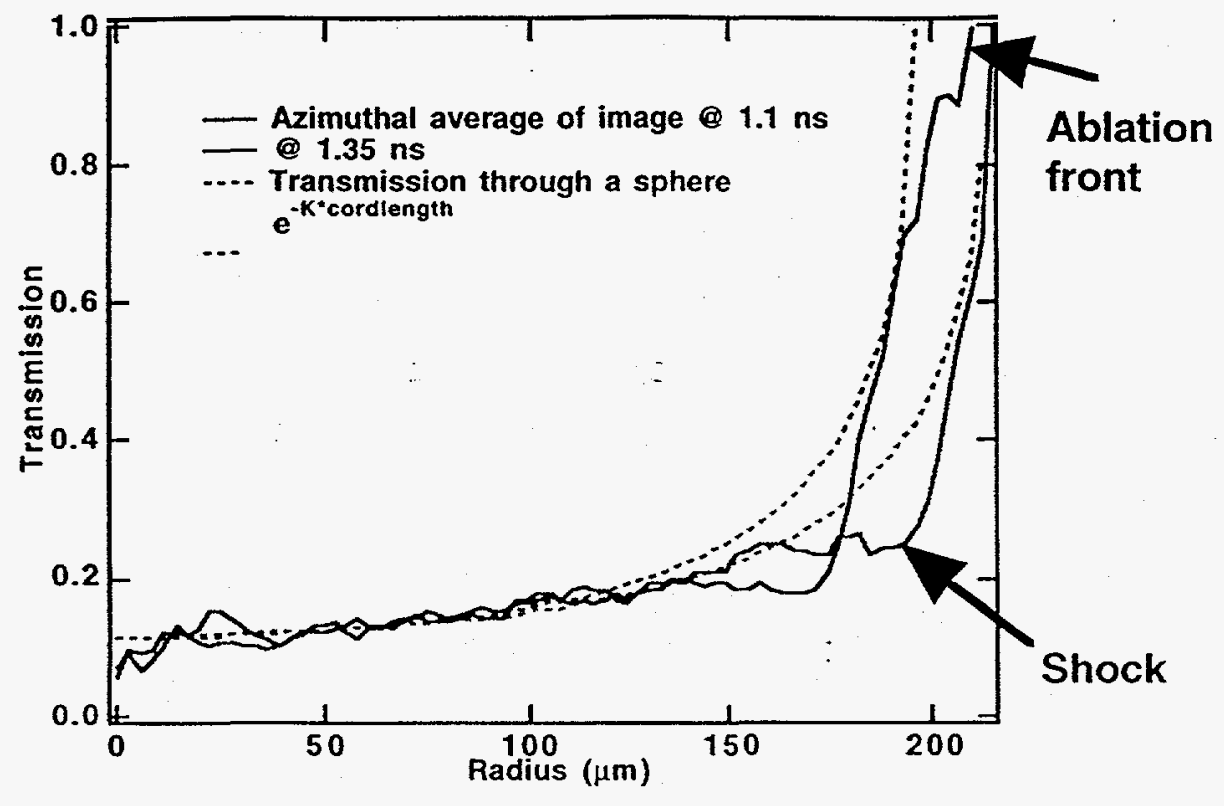

Figure 5. Azimuthal average of backlit images of a foam witness ball. The two curves are from images at two times and the reduced transmission due to shock compression is visible. The rapid increase in transmission corresponding to the rear of the shock before the ablation front is also visible.

\section{Planar Rayleigh-Taylor experiments ${ }^{7,8,910}$}

A more fundamental question relating to the hydrodynamics of implosions is the nature of the RayleighTaylor instability in systems accelerated by ablation. We have quite a large group of experiments which relate to this fundamental instability in a planar geometry. In these experiments the observable is the modulation in transmission of a large area backlighter which corresponds to modulations in optical depth of a foil; as the opacity to $\mathrm{keV} x$-rays is constant throughout the foil, the modulation in transmission is due to areal density modulations in the foil. The modulations arise from (usually) sinusoidal ripples initially present on the surface of the foil; when the foil is accelerated by ablation, the ablation surface is RayleighTaylor (RT) unstable and the modulations are expected to grow exponentially until the amplitude is larger than $10 \%$ of the wavelength and then to become nonlinear, seeding higher harmonics of the initial perturbation and eventually saturating. The foil in the RT experiments may be accelerated either by direct laser ablation or by $x$-ray ablation. The experimental setup for the direct drive case and a typical sequence of images are shown in Figure 6. In this experiment, we used uranium for the backlighter. The uranium spectrum shown in Figure 1 shows a great deal of energy below $1 \mathrm{keV}$; nearly all of this energy would be deposited in the foil and would presumably be sufficient to heat the foil and affect the RT growth. Therefore, a prefilter of $12 \mu \mathrm{m}$ of Be was interposed between the backlighter target and the driven foil, which absorbed most of the $x$-ray energy below about $1 \mathrm{keV}$. (This is the only experiment for which this proved necessary.) The optical depth of the $20 \mu \mathrm{m}$ thick $\mathrm{CH}_{2}$ foil to the remaining uranium spectrum is 1 . Alternative $\mathrm{K}$-shell backlighters at this photon energy have a significant bound-free continuum contribution that reduces the optical depth for a $\mathrm{CH}_{2}$ foil, and hence the modulation in optical depth for a given amplitude.

The sequence of images shows the modulation at the $30 \mu \mathrm{m}$ fundamental frequency quite clearly, and the modulation increases throughout the sequence until the last frame, when foil decompression and saturation of amplitude growth combine to reduce the measured modulation in optical depth. Structures are also visible which appear to run perpendicular to the imposed perturbation; these are modulations due to the drive laser, which, even though smoothed to $<10 \%$ nonuniformity, still has sufficient structure to modulate the foil, and these modulations grow just as preimposed modulations do. It may be noted that the backlighter illuminates 
the lower part of the foil in the earliest frame, progressing downward until in the latest frame it illuminates the upper part of the foil. This is the effect of parallax as the images are formed by lower and lower pinholes in the array. For this reason, the backlighter must be large enough to overfill the area that must be illuminated when gated MCP cameras are the diagnostic.

Indirectly driven planar RT experiments on Nova use a very similar setup, except that the single drive laser beam is replaced by a hohlraum heated with 8 beams to provide the $x$-ray drive. These experiments use bromine doped $\left(\mathrm{C}_{50} \mathrm{H}_{47} \mathrm{Br}_{2.7}\right)$ foils to mismatch the absorption of the foil to the $x$-ray drive spectrum and reduce the foil preheat (and hence increase RT growth). The foils must also be significantly thicker, $\sim 60 \mu \mathrm{m}$ as compared to the direct drive $20 \mu \mathrm{m}$, in order not to bum through during the 2-3 ns drive period. Therefore, it was necessary to choose a higher hv backlighter for these experiments; we used the L-bands of Mo and Rh.
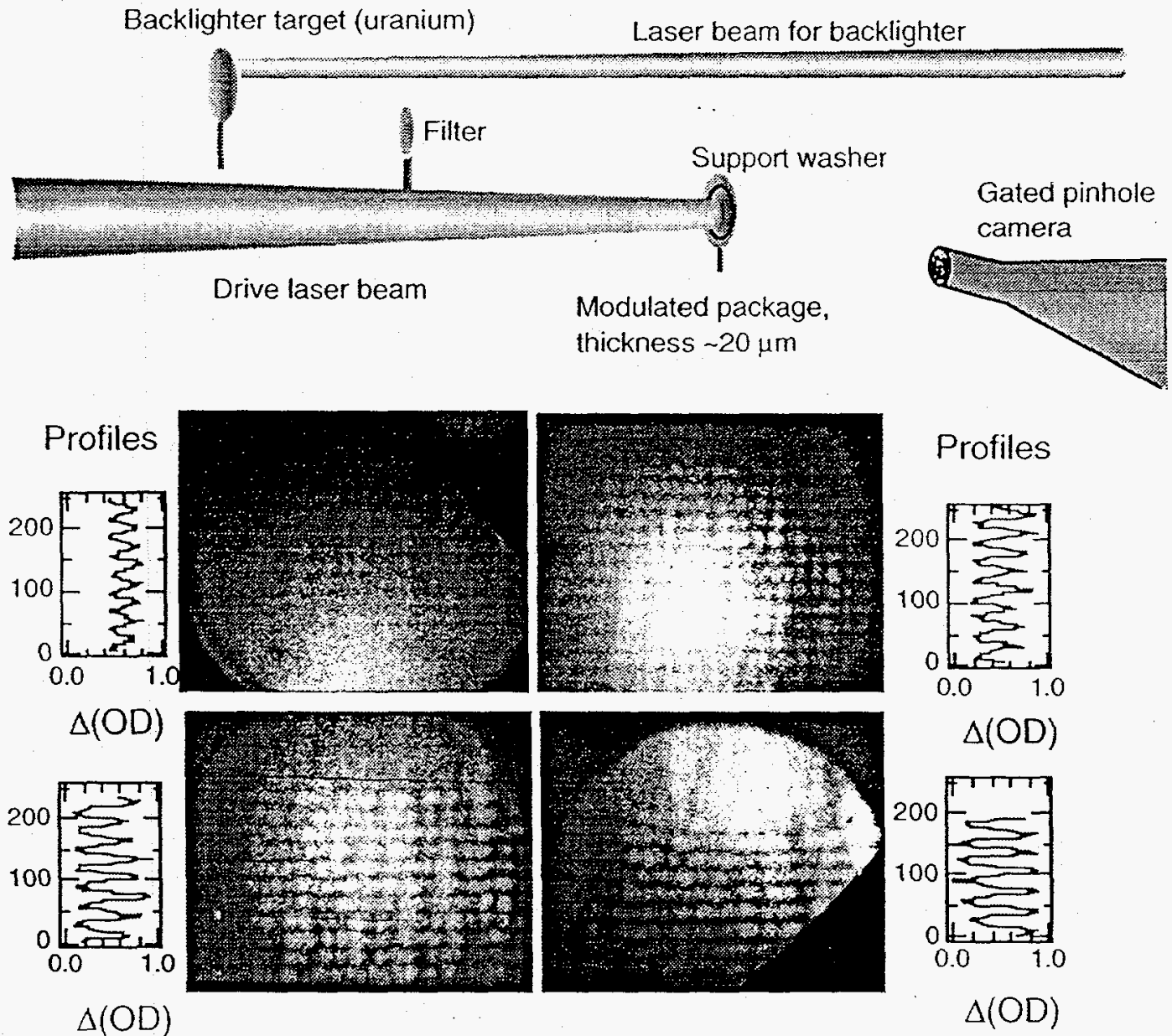

Profiles
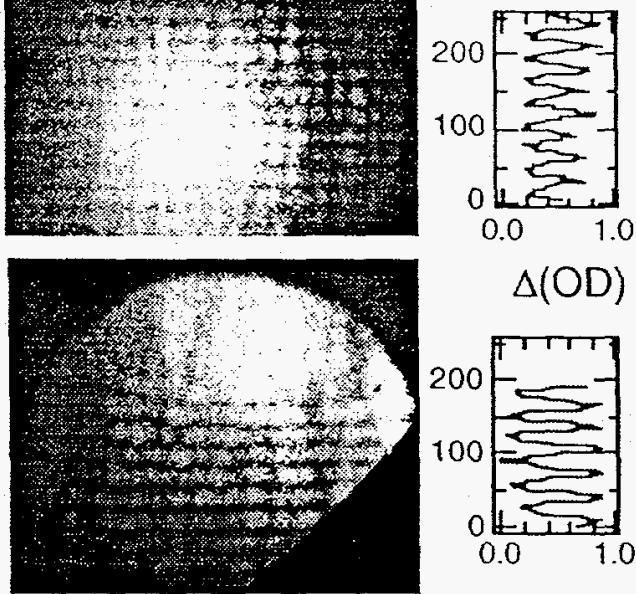

$\triangle(O D)$

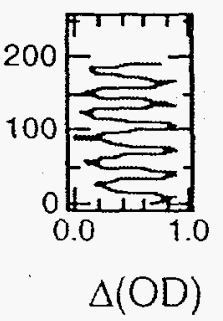

Figure 6. Experimental setup for direct-drive planar Rayleigh-Taylor experiments. The images are (left to right and top to bottom) at $t=1.0,1.5,2.0$, and $2.5 \mathrm{~ns}$ after the start of the drive laser pulse. The size of each image is $700 \mu \mathrm{m}$. The initial wavelength was $30 \mu \mathrm{m}$ and the initial amplitude $0.25 \mu \mathrm{m}$.

\section{Planar laser imprint"}

It is also possible to backlight solid density targets at many optical depths to the backlighter photons, if the backlighter source is sufficiently bright. This is a useful approach in experiments such as the RT experiments described above, where the modulation in optical depth is the measured quantity and the depth of areal 
density modulation measured is required to be small. Then the larger the opacity, the larger the perceived modulation in transmission for a given surface (or areal density) modulation. This is particularly desirable if one wishes to study the imprint of laser structure on a foil prior to RT growth. In this experiment the surface modulations are likely to be only a few tenths of microns, so to produce signals similar in modulation to those in Figure 6 would require 2-3 optical depths/micron in the target. We find a backlighter of sufficient brightness in an $\mathrm{x}$-ray laser; a suitable target for the foil is made of $\mathrm{Si}$, and at the $\mathrm{Y} \mathrm{x}$-ray laser wavelength $(15.5 \mathrm{~mm}$ or $80 \mathrm{eV})$, the cold $\mathrm{Si}$ attenuation is $2.1 / \mu \mathrm{m}$. The backlighter brightness $\left(3-4 \mathrm{~mJ}\right.$ in 200 ps or $10^{17}$ $\left.\mathrm{W} / \mathrm{cm}^{2} / \AA / \mathrm{sr}\right)^{12}$ limits the thickness of the Si sample to 3-4 $\mu \mathrm{m}$. The experimental setup is quite different from those described previously and is shown in Figure 7. An x-ray laser monitoring system is eliminated from the diagram for simplicity. We use multilayer optics to relay the image of the $x$-ray laser at sufficient magnification to illuminate a $100 \mu \mathrm{m}$ square area of the foil, and to image the Si foil onto an $x$-ray CCD. The imaging multilayer optics have a resolution near the optical axis of $\sim 1 \mu \mathrm{m}$.
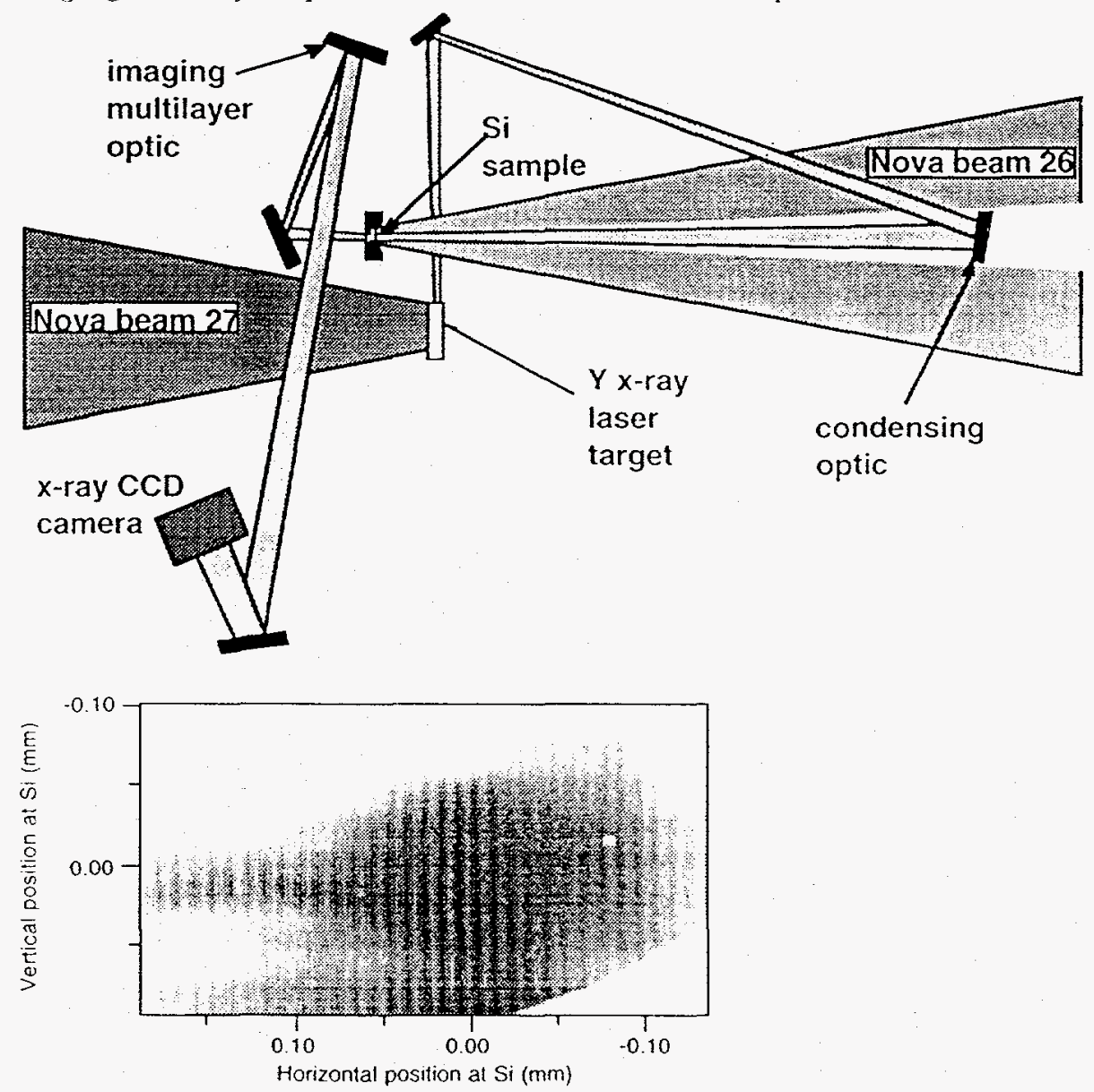

Figure 7. Experimental setup for $x$-ray laser backlighting. We use the $x$-ray laser to image the modulation from laser imprint on a $S$ i foil. An image of a grooved, undriven Si foil shows the resolution of the system and the uniformity of the $x$-ray laser backlighter. 
To test the $x$-ray laser backlighting system, we imaged a patterned, undriven, Si foil $4.1 \mu \mathrm{m}$ thick. 'The grooves were $5 \mu \mathrm{m}$ wide $(100 \mathrm{lp} / \mathrm{mm})$ and $0.24 \mu \mathrm{m}$ deep. The resulting modulation is shown in Figure 7; the modulation agrees with the predicted modulation in optical depth for cold $\mathrm{Si}$, and shows that there are no large modulations in the $x$-ray laser backlighter on a spatial scale of $<10 \mu \mathrm{m}$. We then used the setup to image a foil driven with a low intensity $\left(5 \times 10^{12} \mathrm{~W} / \mathrm{cm}^{2}\right)$ of $0.35 \mu \mathrm{m}$ light from a beam smoothed with a RPP; the $\mathrm{x}$ ray laser produces a pulse $300 \mathrm{ps}$ long which was timed to arrive with the leading edge at the end of the. shock transit time for this foil ( $270 \mathrm{ps}$ after the start of the drive). The resulting image is in Figure 8 ; the modulation in optical depth is clear across the illuminated area of the sample.

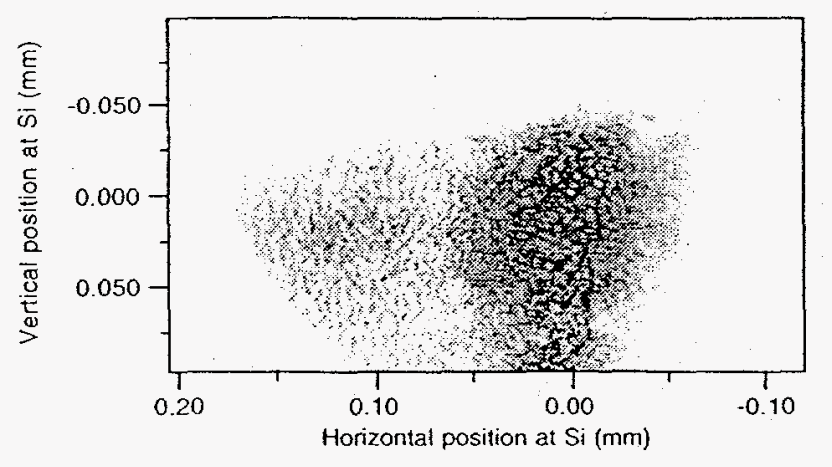

Figure 8. An image of a foil driven with a $0.35 \mu \mathrm{m}$ laser beam smoothed with a RPP shows modulation in optical depth due to laser imprint.

$$
\text { Supemova }{ }^{13},{ }^{14}
$$

The effect of the RT instability is not limited to implosion hydrodynamics, but is also important on a somewhat larger scale in the evolution of a supernova. A supernova has strong density gradients at the interfaces between various layers, which are RT unstable. If the hydrodynamic processes fundamental to a supernova explosion could be scaled to a laboratory experiment, the ability to test the tools for theoretical understanding of supernovas would be advanced significantly. For RT growth in the nonlinear regime (amplitude* wavenumber $>1$ ), a perturbation grows at the rate of its terminal bubble velocity, $u_{B}=a(g \lambda)^{12}$, where $g$ is the acceleration of the interface, $\lambda$ is the wavelength, and $a=0.36$. This suggests that there is $a$ characteristic time scale to the growth process, $\tau=\lambda / u_{B} \approx(\lambda / g)^{42}$. Then if a hydrodynamic system is transformed from supemova to lab, $\lambda_{S N}=a_{1} \lambda_{1}$, and $g_{S N}=a_{2} g_{1}$, the time must scale as $\tau_{S N}=\left(a_{1} / a_{2}\right)^{3 / 2} \tau_{1}$. For a Type II, $25 \mathrm{M}_{\text {sun }}$ supernova, the spatial scale ${ }^{15}$ is $10^{10} \mathrm{~cm}$, the acceleration is $10^{3} \mathrm{~cm} / \mathrm{s}^{2}$, and the time scale is $\sim 10^{4} \mathrm{~s}$. For the lab experiment, the spatial scale is $10^{-4} \mathrm{~cm}$ giving $a_{1}=10^{14}$ and the acceleration is $10^{14} \mathrm{~cm} / \mathrm{s}^{2}$ giving $a_{2}=10^{-11}$, which requires that the time scale in the lab experiment must be $10^{4} \mathrm{~s} /\left(10^{25}\right)^{1 / 2}$, or about $1 \mathrm{~ns}$, a very typical time for these experiments. The other experimental parameters (which would not scale), of pressure, density, mode number. (area/wavelength), and perturbation amplitude/wavelength, are very similar between a supernova and Nova experiment. We show in Figure 9 predicted pressure and density profiles at various times for such a supernova; by $t=1000 \mathrm{~s}$ in the supernova evolution the pressures and densities are achievable on Nova experiments.

A Nova experiment that would mimic the density gradients present in the layers of a supernova might have a copper foil pushing a plastic layer: Both the $\mathrm{Cu}$ and the $\mathrm{CH}$ must be sufficiently thick not to bum through nor to limit hydrodynamic growth of the perturbations during the experiment; this places restrictions on the backlighting. We have begun experiments on Nova to investigate hydrodynamic systems relevant to 
supernovas. The first set of experiments tracked the shocked interface between a $12 \mu \mathrm{m} \mathrm{Cu}$ foil and a $500 \mu \mathrm{m}$ thick $\mathrm{CH}$ tamper. With such thick foils, we use the $\mathrm{K}$-shell lines (mostly $\mathrm{He}-\alpha$ at $6.7 \mathrm{keV}$ ) of $\mathrm{Fe}$ as a backlighter. A typical image is shown in Figure 10.
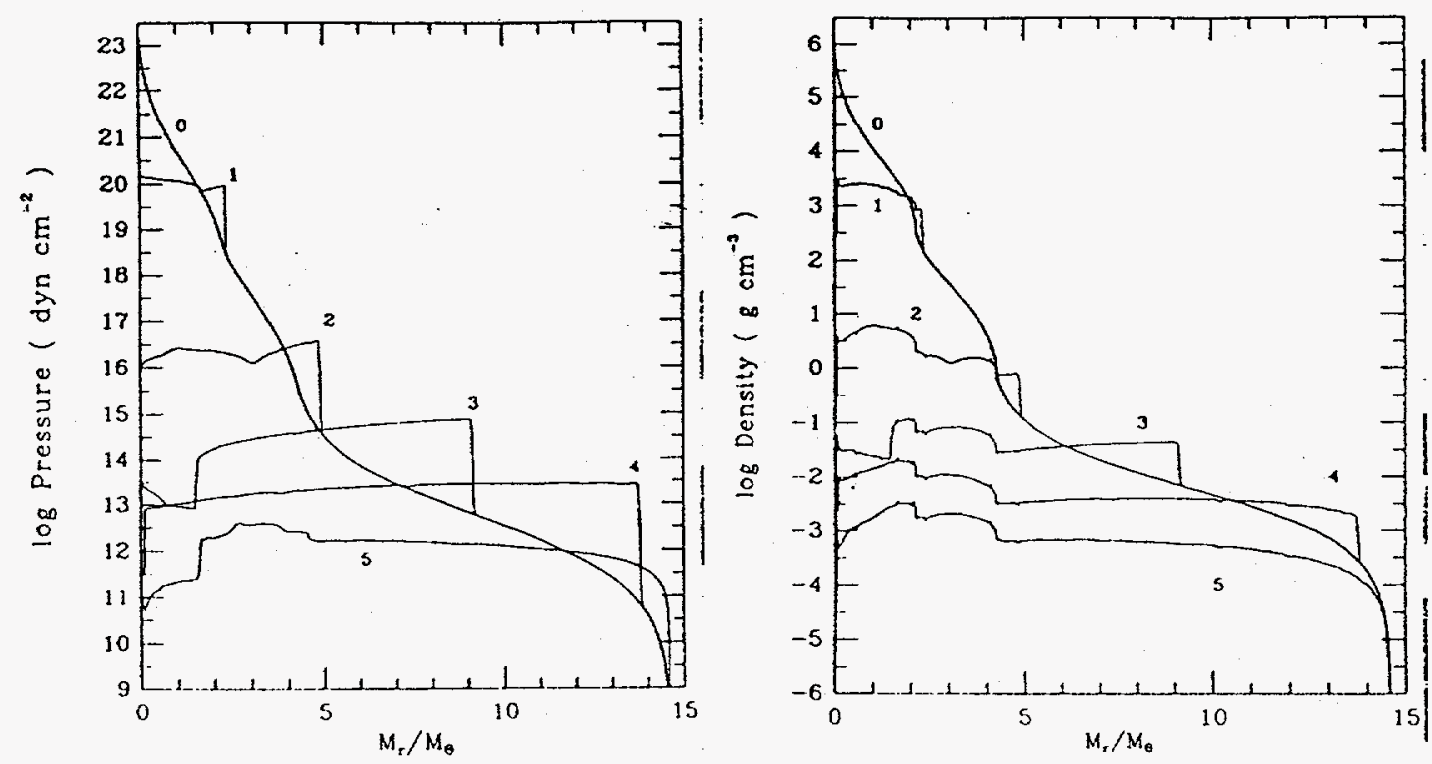

Figure 9. Predicted supemova evolution ${ }^{16}$; pressures achievable in Nova experiments are about $10^{13} \mathrm{dyne} / \mathrm{cm}^{2}$ and densities are about $10 \mathrm{~g} / \mathrm{cm}^{3}$. The curves are at different times: $0 \mathrm{t}=0,1 \mathrm{t}=9 \mathrm{~s}, 2 \mathrm{t}=170 \mathrm{~s}, 3 \mathrm{t}=1000 \mathrm{~s}, 4 \mathrm{t}=3300 \mathrm{~s}$, and $5 \mathrm{t}=7500 \mathrm{~s}$.

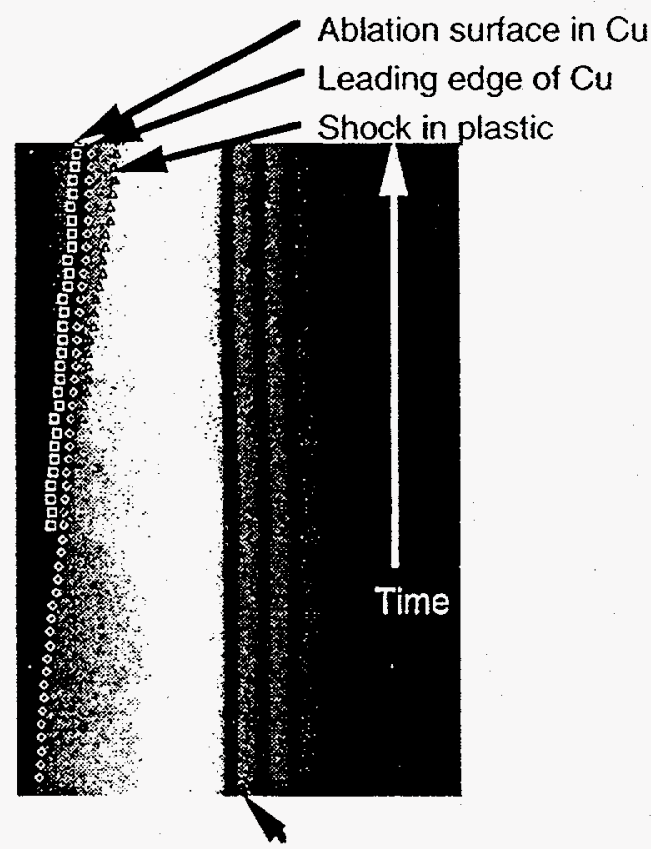

Alignment grid shadow

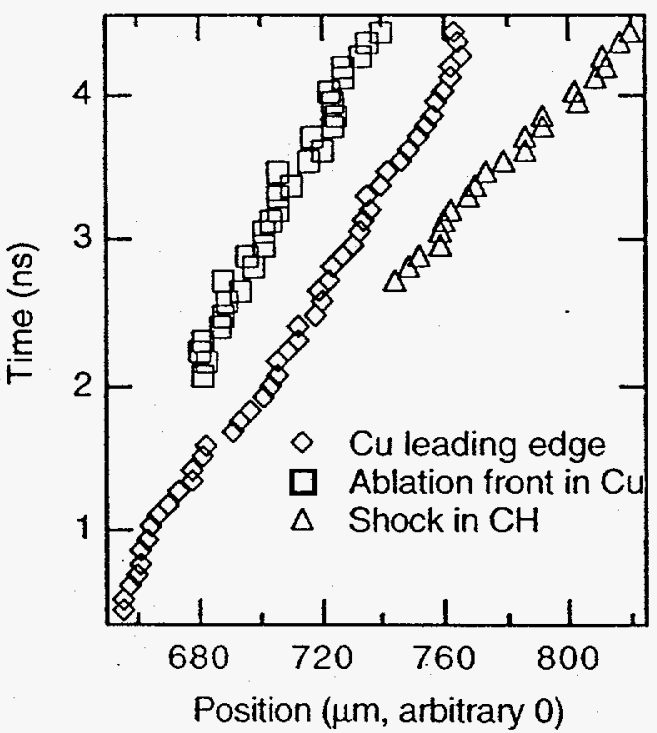

Figure 10. Streak camera image of shock and ablation surfaces propagating in a $\mathrm{Cu} / \mathrm{CH}$ interface. The positions extracted from the image are shown at right. 


\section{Experiments with point backlighters ${ }^{17}$}

We have also done experiments on Nova with a different kind of $x$-ray source, that from a laser-produced plasma which is very small. We used these point backlighters without any other imaging diagnostics; the spatial extent of the point backlighter determines the spatial resolution of the system. They have several advantages: first, the laser beam at best focus irradiates the sample at $\sim 10^{16} \mathrm{~W} / \mathrm{cm}^{2}$ and can produce backlighters in the 8-11 keV range ${ }^{18}$; second, the illumination of the backlit sample is uniform over a large area; and third, the resolution accessible with a fiber as the target is comparable to that of a pinhole camera. However, there are also disadvantages, the most significant being the source size increases with time (at about $100 \mu \mathrm{m} / \mathrm{ns}$ ), so the pulses must usually be short. It is also difficult to get the multiple frame twodimensional imaging (as in the gated MCP pinhole camera) which is so useful in hydrodynamics experiments. We show a point backlighting resolution test experimental setup and result in Figure 11.
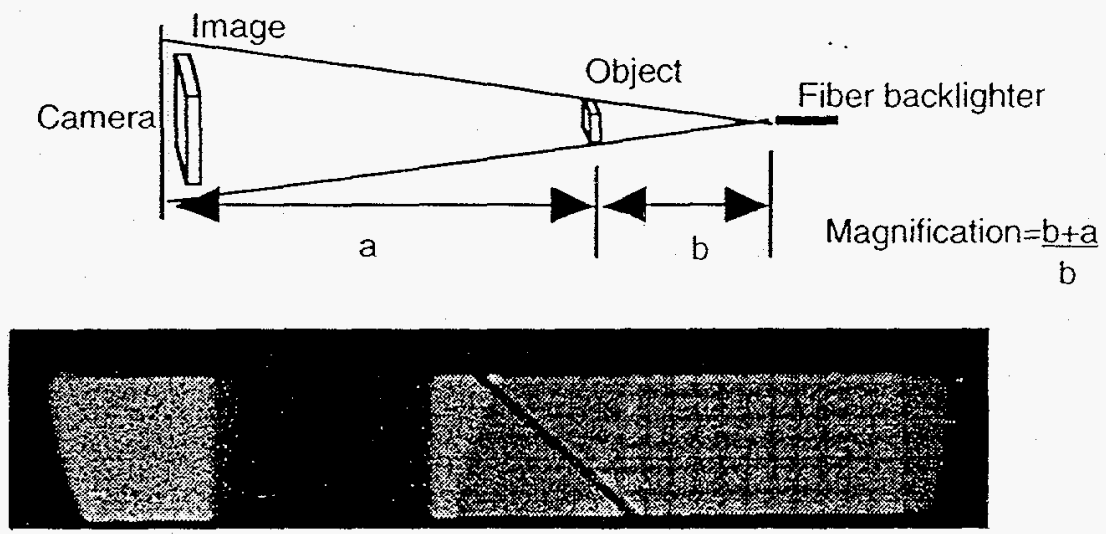

Figure 11. Point backlighter geometry and image of a resolution grid. The backlighter was a round Zn fiber 14 $\mu \mathrm{m}$ in diameter, the pulse duration was $100 \mathrm{ps}$ and the magnification was 18 .

\section{Summary}

We have presented results from some of the many experiments on Nova that employ $x$-rays from laser produced plasmas to backlight dense plasmas in conditions relevant to ICF (and astrophysics). On Nova we use a significant fraction of the laser energy (10-20\%) to achieve bright, large area, high hv backlighter spots with a duration of several nanoseconds. Typically we image foils, capsules, or solid balls with gated microchannel plate pinhole cameras with spatial resolution of $\sim 10 \mu \mathrm{m}$ and temporal resolution of 100 ps. Much better spatial resolution $(\sim 1 \mu \mathrm{m})$ may be achieved in certain specialized applications by the use of an $x-$ ray laser as a backlighter; the very high brightness of an $x$-ray laser compensates for the high opacily of most cold dense plasmas to such low energy radiation and allows backlighting of systems with many ( 10 for our experiment) optical depths. Hydrodynamics of ICF and astrophysical systems may be examined by measuring Rayleigh-Taylor growth in planar configurations; drive symmetry in hohlraums may be addressed by measuring nonuniform shock propagation in low density foams; and even implosions may be backlit to determine density parameters during acceleration of the capsule shell.

\section{Acknowledgments}

We would like to thank J.D.Kilkenny, P.M.Bell, S.V.Weber, J.P.Knauer, L.J.Suter, and R.L.Kauffman for very useful discussions. This work performed under the auspices of the U.S. Department of Energy by Lawrence Livermore National Laboratory under contract No. W-7405-ENG-48. 


\section{References}

' O.L.Landen et al., Ultrahigh- and High-Speed Photography, Photonics, and Videography 932002 (1993).

${ }^{2}$ J.D.Kilkenny, Phys. Fluids B 2, 1400 (1990).

${ }^{3}$ L.Koppel and J.Eckels, UCRL-79781 (1977).

${ }^{4}$ D.L.Matthews et al., J. Appl. Phys. 54, 4260 (1983).

${ }^{5}$ O.L.Landen et al., Jour. Quant. Spectr. Rad. Trans., (to be published).

${ }^{6}$ P.Amendt et al., Rev. Sci. Instrum. 66, 785 (1995).

${ }^{7}$ S.G.Glendinning et al., Phys. Rev. Lett. 69, 1201 (1992).

${ }^{8}$ B.A.Remington et al., Phys Rev. Lett. 73, 545 (1994).

${ }^{9}$ B.A.Remington et al., Phys. Fluids B 5, 2589 (1993).

${ }^{10}$ B.A.Remington et al., Phys Fluids B 4, 967 (1992).

${ }^{11}$ M.H.Key, these proceedings

${ }^{12}$ R.Cauble et al., Phys. Rev. Lett. 74, 3816 (1995).

${ }^{13}$ E.Muller, F.Fryxell, and D.Arnett, Astron. Astrophys. 251, 505 (1991).

${ }^{14}$ M.Herant and S.E.Woosley, Ap. J. 425, 814 (1994).

${ }^{15}$ Woosley et al., private communication.

${ }^{16}$ Shigeyama and Nomoto, Ap. J. 360, 242 (1990).

${ }^{17}$ T.S.Perry et al., Phys. Rev. Lett. 67, 3784 (1991).

${ }^{18}$ J.D.Molitoris et al., Rev. of Sci. Inst. 63, 5104 (1992). 